\title{
Etude au laboratoire des effets d'un insecticide naturel extrait du neem (Azadirachta indica A. Juss) sur Glossina fuscipes fuscipes Newstead, 1910 (Diptera : Glossinidae)
}

\author{
P.B. Makoundou ${ }^{1 *}$, D. Cuisance ${ }^{2}$, G. Duvallet ${ }^{2}$, P. Guillet ${ }^{1}$
}

MAKOUNDOU P.B., CUISANCE D., DUVALLET G., GUILLET P. Etude au laboratoire des effets d'un insecticide naturel extrait du neem (Azadirachta indica A. Juss) sur Glossina fuscipes fuscipes Newstead, 1910 (Diptera : Glossinidae). Revue Elev. Méd. vét. Pays trop., 1995, 48 (4) : $339-345$

Dans la perspective d'une lutte contre les glossines avec sa prise en charge par les communautés rurales, nous avons testé l'Azatin $\mathbf{E} \mathbf{C}^{\circledR}$, produit à base d'extraits de graines de neem (Azadirachta indica A.' Juss). Les tests sont effectués sur Glossina fuscipes fuscipes, par applications topiques et par contact tarsal. L'application topique d'Azatin $\mathrm{EC}^{\oplus}$, formulation contenant $30 \mathrm{~g}$ d'azadirachtine par litre, provoque une faible mortalité qui ne se manifeste qu'aux doses élevées $(2,61 \mu \mathrm{g}$ d'azadirachtine/mouche) chez les jeunes mâles (DL50 = $0,747 \mu \mathrm{g})$ et les femelles âgées et gravides $(\mathrm{DLS0}=\mathbf{2 , 5 1 6} \mu \mathrm{g}$ ). La productivité (pupes/femelle) est abaissée de 4,5 lois pour les doses élevées et ensuite le poids des pupes est significativement plus bas en début de pupaison mais n'évolue pas différemment de celui des témoins. Le taux d'éclosion est significativement abaissé aux doses supérieures à $\mathbf{0 , 2 6 1} \mu \mathrm{g} / \mathrm{mouch}$. Des difficultés de vol et de sondage (piqûre) sont notées à partir de $0,261 \mu \mathrm{g} / \mathrm{glossine}$, semblant traduire une perturbation de la physiologie musculaire. Par contre, le contact tarsal sur un tissu traité à une dose de $3,9 \mathrm{~g} / \mathrm{m}^{2}$ d'azadirachtine n'a aucun effet sur la mortalité, le taux d'éclosion et la productivité pour les trois temps de contact étudiés (1, 2 et 3 min). En revanche, un fort effet répulsif est observé après un badigeonnage de l'oreille de l'hôte nourricier avec une solution à $0,3 \mathrm{~g} / \mathrm{l}$ d'azadirachtine. A cette concentration, 70 p. 100 des glossines ne se nourrissent pas et ce chiffre atteint $90 \mathrm{p}$. 100 à la concentration de $3 \mathrm{~g} / \mathrm{l}$. En fonction des effets observés chez cet insecte hématophage, les applications envisageables sur le terrain sont discutées, soulignant la possibilité de mettre à profit cet effet répulsif pour protéger le bétail dans les zones à risque.

Mots-clés : Glossina - Azadirachta indica - Lutte anti-insecte - Acadirachtine - Phagorépulsif - Insecticide - Pupe - Application locale.

\section{INTRODUCTION}

Les problèmes économiques actuels de l'Afrique font que l'importation des insecticides de synthèse pour lutter contre les insectes vecteurs constitue une lourde charge financière. Celle-ci pourrait être allégée par l'utilisation de produits insecticides naturels disponibles localement. L'utilisation de tels produits pourrait même permettre la participation des communautés rurales concernées par la lutte et présenter des avantages sur le plan écologique.

1. ORSTOM, Laboratoire des Insectes nuisibles, BP 5045, 34032 Montpellier Cedex, France.

* Adresse actuelle : c/o D. Cuisance.

2. CIRAD - EMVT, BP 5035, 34032 Montpellier Cedex, France.

Reçu le 13.7.95, accepté le 8.3.96.
Les extraits d'un arbre tropical, le neem (Azadirachta indica A. Juss), ont montré des propriétés insecticides sur divers insectes, notamment sur Musca autumnalis (6) et sur plusieurs insectes d'intérêt agricole tels que Ostrinia nubialis (12), Dysdercus koenigi (8), Liriomysa trifolii (17), Bemisia tabaci (13). La toxicité de cet arbre sur les insectes est due à une substance qui est l'azadirachtine. Relativement peu de recherches ont été faites sur la toxicité des substances insecticides naturelles contre les insectes d'intérêt médical et vétérinaire. L'azadirachtine a montré des propriétés insecticides sur les moustiques (3, 18). Sur les glossines, on observe une très forte mortalité en trois jours de Glossina fuscipes fuscipes après leur introduction dans des cages dont le tulle a été sommairement imprégné d'extraits de graines de neem ( $G$. Duvallet, observation non publiée).

La tendance actuelle est de promouvoir la création d'organisations villageoises dans le domaine agricole, et de groupements d'intérêts pastoraux dans le domaine de l'élevage afin de développer leur participation à la réalisation des actions de lutte par l'utilisation de pièges et d'écrans attractifs imprégnés d'insecticides. Cela incite les chercheurs à trouver des solutions locales aux problèmes des parasitoses.

Les trypanosomoses, dont les glossines sont les principaux agents vecteurs, demeurent préoccupantes en raison de la reviviscence de nombreux foyers et de leur impact sur la santé humaine et animale et, en conséquence, sur l'économie des pays africains. Dans ce cadre, nous avons testé la toxicité de l'azadirachtine sur les glossines, notamment sur Glossina fuscipes fuscipes.

\section{MATERIEL et METHODES}

Les glossines proviennent de l'élevage CIRAD-ORSTOM de Montpellier. Les individus sont maintenus dans des cages de type "Roubaud" (14 x 8,5 x $5 \mathrm{~cm}$ ) à raison de 25 à 30 individus par cage. Les mouches sont nourries cinq jours par semaine en plaçant les cages sur les oreilles d'un lapin. Les lots sont maintenus après traitement à une température comprise entre 23 et $25^{\circ} \mathrm{C}$ avec une humidité relative de 70 à 80 p. 100. La photopériode est de $12 \mathrm{~h}$ de jour et de $12 \mathrm{~h}$ de nuit (éclairage artificiel). Les extraits de neem ou Azatin $\mathrm{EC}^{\circledast}$ ont été fournis par la société Calliope. Le produit est une émulsion contenant 


\section{P.B. Makoundou D. Cuisance G. Duvallet P. Guillet}

10 p. 100 d'extraits de neem dont 3 p. 100 d'azadirachtine. Nous avons testé la toxicité du produit pour les glossines, d'une part en applications topiques et d'autre part en contact tarsal.

\section{Applications topiques}

Le produit est dilué dans un solvant composé de $50 p$. 100 d'acétone et de 50 p. 100 de dioctylphtalate. La dose d'insecticide est calculée sur la base du produit actif (l'azadirachtine). Une micro goutte de $0,87 \mathrm{ml}$ de solution est appliquée sur la face dorsale du thorax de chaque mouche, délivrant respectivement $2,61,0,261,0,0261 \mu \mathrm{g}$ d'azadirachtine par mouche. Les glossines sont traitées individuellement avec un micro-applicateur de type Arnold. Avant chaque manipulation, elles sont nourries puis transportées vers la salle de test dans laquelle elles sont anesthésiées avec du $\mathrm{CO} 2$ et traitées immédiatement. Les tests sont d'abord effectués avec des femelles âgées et gravides, ainsi que de jeunes mâles pour évaluer l'effet de l'azadirachtine sur la mortalité. Tous les jours, les glossines mortes sont comptées et retirées des cages. Lorsqu'on dispose d'au moins trois doses donnant des mortalités supérieures à 0 et inférieures à 100 p. 100 dont une au moins entre 0 et 50 p. 100 , les résultats sont analysés par la méthode Log Probit avec un logiciel spécialisé (Probit Analysis Program, version 3.1, CNRSUSTL Montpellier). Ensuite, les tests sont effectués sur de jeunes femelles pour suivre l'impact du traitement sur la reproduction. Avant celui-ci, elles sont accouplées à l'âge de 2 ou 3 jours avec des mâles qui ont entre 7 et 10 jours (âges connus comme le plus favorable à la fécondation). Elles sont mises en présence des mâles pendant $72 \mathrm{~h}$, puis les sexes sont séparés. Chaque jour, les pupes sont récoltées et comptées. Elles sont pesées individuellement tous les 7 jours pendant toute la durée de pupaison afin de suivre l'évolution de leur poids. De même, le nombre d'imagos émergeant est compté pour évaluer le taux d'éclosion. En dernier lieu, la productivité $P$ pour chaque lot est calculée :

$$
\mathrm{P}=\frac{\text { Nombre de pupes }}{\text { Nombre de femelles reproductrices } \mathrm{x} \text { jour }}
$$

Le nombre de femelles reproductrices $x$ jour est obtenu en additionnant le nombre de mouches vivantes chaque jour à partir de la première larviposition (10).

\section{Contact tarsal forcé}

Nous avons imprégné un tissu bleu (coton 33 p. 100 polyester 63 p. 100) identique à celui utilisé dans les campagnes de lutte à une concentration de $3,9 \mathrm{~g} / \mathrm{m}^{2}$ d'azadirachtine. Les mouches non anesthésiées sont mises en contact avec le tissu traité par les tarses en les maintenant par les ailes pendant des temps variables : 1,2 et $3 \mathrm{~min}$. Le témoin est constitué par un lot de mouches manipulées de la même façon mais sur du tissu non traité. Les tests sont effectués sur des femelles âgées et gravides ainsi que sur de jeunes femelles. Les paramètres suivis sont les mêmes que ceux des applications topiques.

\section{Contact tarsal spontané}

Des femelles, à jeun depuis 48 à $72 \mathrm{~h}$ et mises par lots de 30 en cage Roubaud, sont nourries sur l'oreille des hôtes nourriciers dont la peau est badigeonnée à différentes concentrations de produit : $3 \mathrm{~g} / \mathrm{l}, 0,3 \mathrm{~g} / \mathrm{l}, 0,03 \mathrm{~g} / \mathrm{l}$ d'azadirachtine. La cage témoin est posée sur l'oreille non traitée. Les cages sont maintenues 10 min sur l'hôte nourricier: Le nombre de femelles qui prennent un repas de sang est compté.

\section{RESULTATS}

\section{Applications topiques}

\section{Mortalité}

Du fait des faibles mortalités observées, seuls deux tests ont pu faire l'objet d'une analyse Probit. Chez les jeunes mäles, l'analyse Probit est faite au cinquième jour (figure 1) de lecture de la mortalité ( $D L 50=0,747 \mu \mathrm{g}$ ) et au septième jour (figure 2) pour les femelles âgées et gravides $(\mathrm{DL} 50=2,516 \mu \mathrm{g})$.

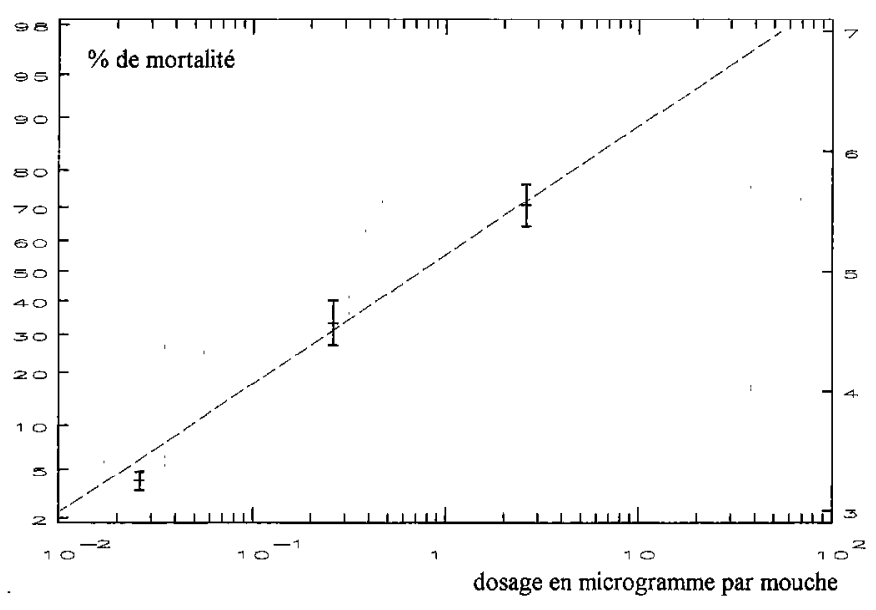

Figure 1: Droite de régression dose/mortalité chez des jeunes glossines mâles au $5^{e}$ jour après applications topiques. 


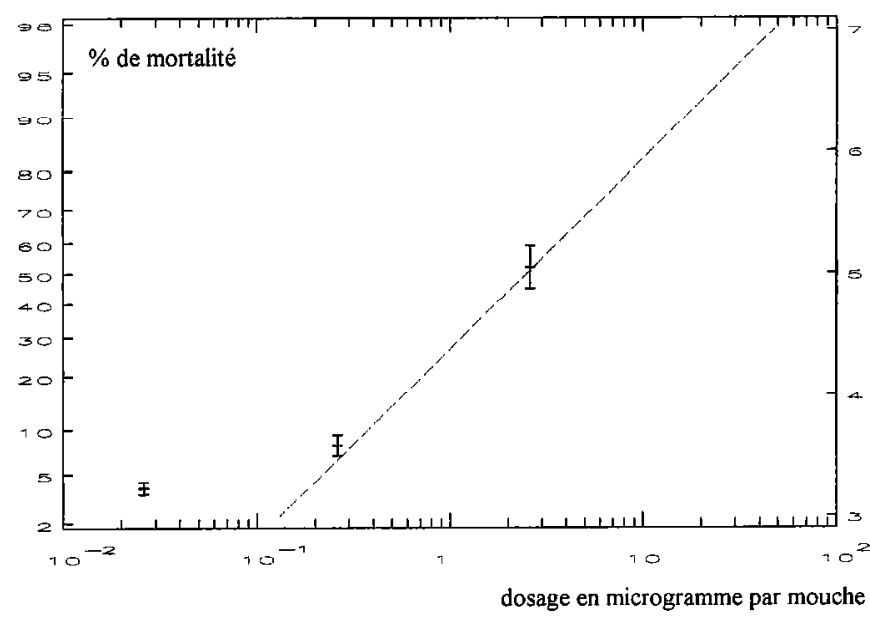

Figure 2 : Droite de régression dose/mortalité chez des glossines âgées et gravides au $7^{\mathrm{e}}$ jour après applications topiques.

Dans les deux cas, la mortalité n'est jamais totale malgré les fortes doses appliquées, ce qui traduit une toxicité assez faible du produit vis-à-vis de Glossina fuscipes fuscipes.

\section{Poids des pupes}

\section{Poids des pupes au jour Jo (tableau I)}

Au moment de la larviposition, l'observation du poids des pupes montre des différences en fonction de la dose d'azadirachtine reçue par comparaison au tot témoin. Les analyses statistiques indiquent une différence significative entre le lot témoin et celui traité à $0,261 \mu \mathrm{g} / \mathrm{mouche}$ ( $\mathrm{t}=6,92 ; \mathrm{H} . \mathrm{S}$.) ainsi que le lot traité à $2,61 \mu \mathrm{g} / \mathrm{mouche}$ $(t=10,32 ; H . S$.$) .$

\section{TABLEAU I}

Poids en mg des pupes de Glossina fuscipes fuscipes à la larviposition en fonction de la dose (applications topiques)

\section{Dose/mouche.}

en $\mu \mathrm{g} \quad$ Témoin $\quad 0,0261 \quad 0,261 \quad 2,61$

\section{Nombre de}

pupes

59

49

45

24

Moyenne
La différence est également significative entre le lot de femelles traitées à $0,0261 \mu \mathrm{g} /$ mouche et le lot traité à $0,261 \mu \mathrm{g} /$ mouche $(\mathrm{t}=5,06 ; \mathrm{S})$. Les effets de l'azadirachtine se traduisent par un poids inférieur des pupes juste après la larviposition à partir de la dose de 0,261 $\mu \mathrm{g} /$ mouche.

\section{Poids des pupes pendant la pupaison}

Dans les conditions normales de laboratoire, la perte de poids des pupes est importante entre le $1^{\text {er }}$ et le $7^{\mathrm{e}}$ jour, et devient ensuite minime jusqu'à l'éclosion (2).

Après constat d'une différence significative du poids juste après la larviposition, les pupes issues du lot témoin et celles issues des lots traités ont des évolutions identiques au cours de la pupaison (figure 3 ). L'azadirachtine n'affecte pas le poids des pupes au cours du développement de ces dernières.

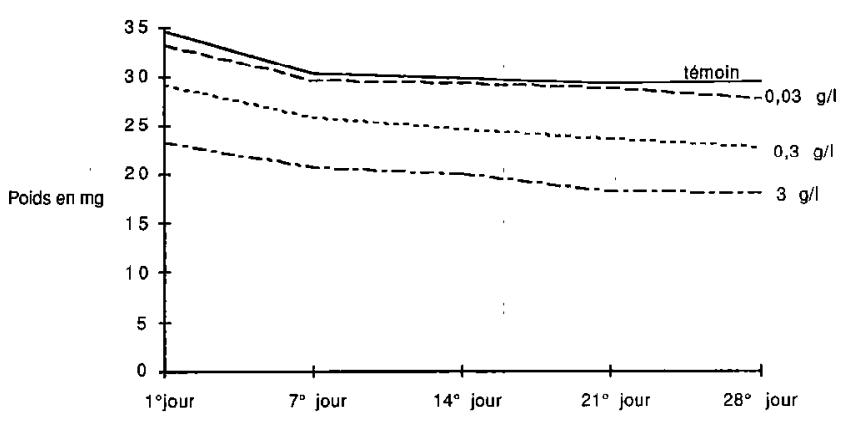

Figure 3: Evolution du poids des pupes dans le lot témoin et les lots traités par application topique sur les femelles reproductrices

\section{Taux d'éclosion (tableau II)}

Les tests statistiques ne montrent aucune différence significative ( $\varepsilon=0,57 ; N$.S.) entre le taux d'éclosion des adultes issus des pupes produites par le lot témoin et celui issu des pupes produites par les femelles traitées à $0,0261 \mu \mathrm{g} /$ mouche. Les pupes émises par les femelles des lots traités à $0,261 \mu \mathrm{g} /$ mouche présentent un taux d'éclosion de 74,47 p. 100 , soit une différence à peine significative par rapport au témoin $(\varepsilon=1,97)$. Pour les femelles ayant reçu la dose de $2,61 \mu \mathrm{g} /$ mouche, le taux d'éclosion est de 50 p. 100 , la différence est hautement significative par rapport au témoin ( $\mathcal{E}=3,08$; H.S.). En revanche, la différence entre les femelles ayant reçu la dose de 0,261 et celles issues des lots traités à 2,61 $\mu \mathrm{g} /$ mouche n'est pas significative $(\boldsymbol{E}=1,53)$. L'effet de l'azadirachtine sur l'éclosion n'est sensible qu'à partir de la dose de $0,261 \mu \mathrm{g} /$ mouche. 
P.B. Makoundou D. Cuisance G. Duvallet P. Guillet

\section{Productivité (tableau II)}

Elle est affectée lorsque les jeunes femelles sont traitées à la dose de $2,61 \mu \mathrm{g} /$ mouche avec une différence significative entre le lot traité $(0,013$ pupe/femelle) et le lot témoin $(0,059$ pupe/femelle) $(\varepsilon=2,99 ; \mathrm{S})$. La productivité du lot de femelles traitées à la plus forte dose d'azadirachtine est 4,5 fois plus faible que celle du lot témoin. L'effet du traitement sur la productivité ne se manifeste qu'à $2,61 \mu \mathrm{g} /$ mouche.

\section{Autres effets}

Nous observons qu'un traitement à l'azadirachtine entraîne une certaine incapacité de vol de Glossina fuscipes fuscipes et une difficulté à remonter la trompe après le repas de sang pour les lots traités à 0,261 et 2,61 $\mu \mathrm{g} /$ mouche. II semble que l'azadirachtine affecte la physiologie musculaire des glossines en particulier au niveau des muscles alaires et ceux du proboscis.

\section{TABLEAU II}

Taux d'éclosion et productivitê par des femelles de Glossina fuscipes fuscipes traitées par applications topiques d'Azatin EC ${ }^{(1)}$

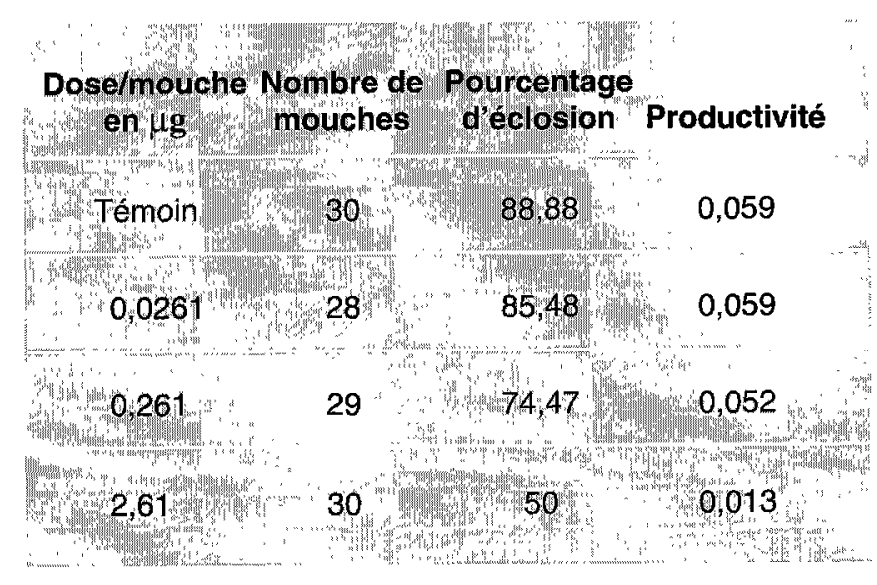

Temps d'observation : 40 jours après la première larvipusition

\section{Contact tarsal}

\section{Contact forcé}

\section{Mortalité}

Aucune mortalité n'a été observée chez les femelles âgées et gravides ainsi que chez les jeunes femelles après le contact sur le tissu traité quel que soit le temps de contact.
TABLEAU III

Taux d'éclosion et productivité des femelles de Glossina fuscipes fuscipes après contact tarsal

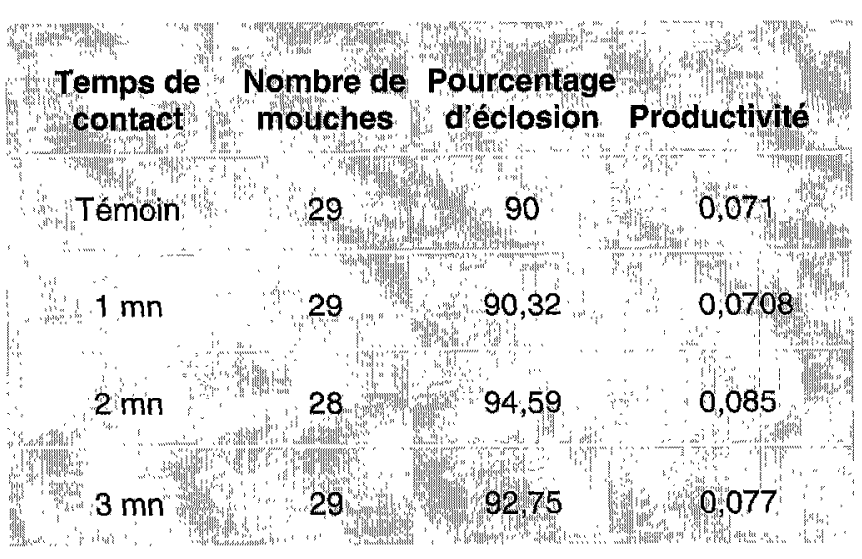

Temps d'observation 31 jours après la première larviposition

\section{Taux d'éclosion (tableau III)}

Les taux d'éclosion chez les lots traités ne montrent aucune différence significative par rapport au lot témoin pour les trois temps de contact $\left(\varepsilon_{1}=0,06\right.$, N.S. ; $\varepsilon_{2}=$ 1,00 , N.S. $; \varepsilon_{3}=0,55$, N.S.).

Productivité (tableau III)

Les conclusions sont identiques à celles du taux d'éclosion et de la mortalité $\left(t_{1}=0\right.$, N.S. $; t_{2}=0,45$, N.S. $; t_{3}$ $=0,22$, N.S.). Le contact tarsal avec l'azadirachtine n'affecte pas la productivité des femelles fécondées.

\section{Contact naturel (tableau IV)}

\section{TABLEAU IV}

Nombre dé femelles âgées et gravides gorgées et non gorgées sur des oreilles de lapins traitées à différentes doses d'Azatin $\mathrm{EC}^{(}$

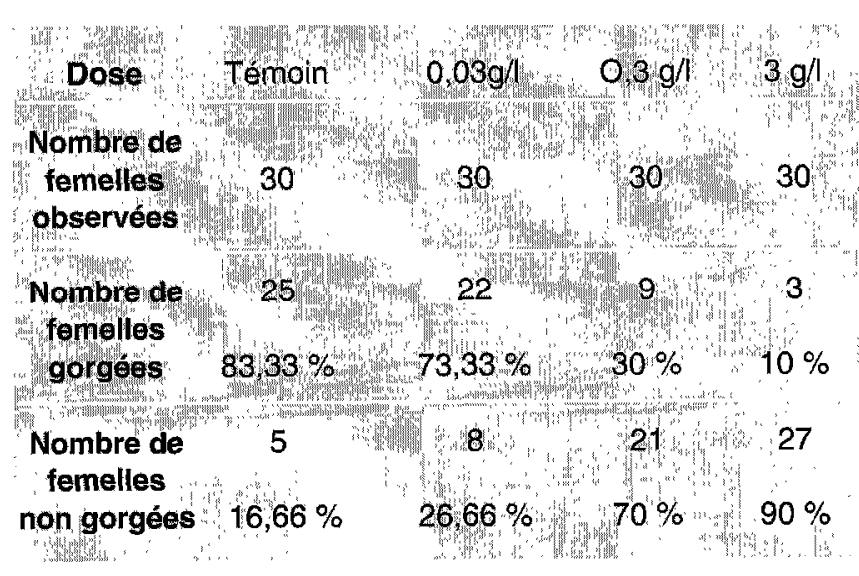


Le nombre de femelles gorgées diminue proportionnellement à la concentration d'azadirachtine badigeonné sur les oreilles du lapin avec une différence hautement significative à la concentration de $0,3 \mathrm{~g} / \mathrm{l}\left(\chi^{2}=17,35\right.$; ddl $=1$; H.S.) et de $3 \mathrm{~g} / \mathrm{l}\left(\chi^{2}=32,41\right.$; ddl = 1; H.S.) (figure 4).

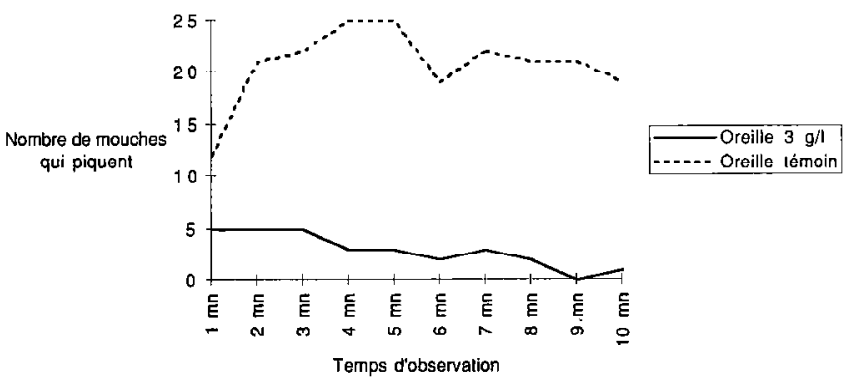

Figure $4:$ Effet phagorépulsif : nombre de femelles qui piquent en fonction du temps d'observation pour la dose de 3 gll appliquée sur la peau de lapin.

\section{DISCUSSION}

\section{Effets insecticides}

\section{Effets sur la mortalité}

Les faibles mortalités obtenues chez les femelles âgées et gravides (classiquement réputées comme les moins sensibles aux insecticides) et sur les jeunes mâles lors d'applications topiques, traduisent une relation dose/mortalité plus faible que celle généralement observée avec les insecticides conventionnels de synthèse. Pour l'endosulfan, le rapport DL90/DL50 est de 2,57 chez les femelles gravides de Glossina fuscipes fuscipes (7) tandis que ce rapport est de 6,63 pour l'azadirachtine. L'utilisation de la formulation testée n'est pas envisageable dans le cas de pulvérisations sur le terrain compte tenu des doses qu'il faudrait appliquer pour obtenir une forte mortalité. L'absence de mortalité ou d'effet knock-down (tel celui observé avec les pyréthrinoïdes) par contact tarsal forcé ne permet pas non plus d'envisager l'utilisation des extraits de neem dans la lutte antiglossines par écrans ou pièges imprégnés.

\section{Effets hormonaux}

Au Ghana, D. Dankwa (non publié) a observé une baisse de la fécondité des femelles et du poids des pupes en nourrissant des femelles de Glossina palpalis palpalis sur les flancs de chèvre traités avec des extraits de feuilles de neem. Nos résultats obtenus en applications topiques sont comparables en indiquant une baisse significative du poids des pupes à partir de la dose de $0,261 \mu \mathrm{g} /$ mouche. II semble donc que le traitement avec les extraits de feuilles ou de graines de neem affecte la productivité et le poids des pupes.

Rembold et coll. (14) ont trouvé des résultats similaires sur Locusta migratoria après injection de l'azadirachtine. Le titrage hormonal a montré une baisse concomitante de l'hormone juvénile et de l'ecdysone ainsi qu'un décalage dans le temps de leur sécrétion chez l'adulte. Par similitude, on peut penser que l'azadirachtine affecte la sécrétion d'hormones chez l'adulte de Glossina fuscipes fuscipes. En effet, les taux d'éclosion sont fortement réduits dans les lots traités à la plus forte dose.

Cette baisse a été également notée par D. Dankwa (non publié) chez Glossina palpalis palpalis après un contact tarsal avec des extraits de feuilles de neem. Bidmon et coll. (1) ont montré que l'azadirachtine se comporte comme un mimétique d'hormone ecdysoïde en inhibant l'émergence des adultes chez Calliphora vicina après injection d'azadirachtine aux larves. Dorn et coll. ont observé le même effet sur Oncopeltus fasciatus (4). Gaaboub et Hayes (6) ont également obtenu des inhibitions d'émergence d'adultes chez Musca autumnalis après une exposition des larves à l'azadirachtine.

\section{Effets neurotoxiques}

On constate que les lots de glossines traitées par des applications topiques sont incapables de voler et ont des difficultés à remonter la trompe après le repas de sang pris sur le lapin. Naqvi (11) a remarqué qu'une mixture de triterpénoïdes provenant de fruits mûrs de neem provoquait une inhibition de la cholinestérase chez Musca domestica. II est possible que le même type d'inhibition se manifeste chez la glossine, expliquant les désordres physiologiques musculaires observés. Dans la nature, la glossine qui ne peut plus voler est condamnée car elle est à la merci des prédateurs (fourmis en particulier) (9). Cet effet "paralysant " se surajoute à la mortalité directe.

II apparaît donc que l'Azatin EC $\mathrm{E}^{\circledast}$ a des effets insecticides sur Glossina fuscipes fuscipes. Mais, ces effets sont trop faibles en général pour envisager l'utilisation de cette formulation sur le terrain, soit par pulvérisation, soit par imprégnation des leurres. Toutefois, Stark et Walter (16) en comparant une autre préparation commerciale, (Margosan- ${ }^{\circledR}$ ) à l'Azatin $\mathrm{EC}^{\circledR}$, ont montré que certains composants de l'huile de neem augmentent significativement l'effet insecticide des préparations commerciales sur Acyrthosiphon pisum (Harris). 


\section{Effet phagorépulsif}

Après avoir badigeonné les flancs de chèvre avec des extraits de feuilles de neem, D. Dankwa (non publié) n'a observé aucun effet répulsif. Contrairement à ces résultats; nous avons remarqué qu'après avoir enduit l'oreille de lapin avec la formulation (essai réalisé avec trois doses différentes : $0,03 \mathrm{~g} / \mathrm{l} ; 0,3 \mathrm{~g} / \mathrm{l} ; 3 \mathrm{~g} / \mathrm{l}$ d'azadirachtine), une proportion très importante de glossines à jeun a refusé de se nourrir (de 70 à 90 p. 100 pour les concentrations les plus élevées). Cet effet phagorépulsif est couramment observé sur les insectes d'intérêt agricole. Ces propriétés sont utilisées en Asie et en Afrique pour protéger les cultures contre les ravageurs. II apparaît donc que l'Azatin $\mathrm{EC}^{\circledR}$ exerce un effet phagorépulsif sur les glossines en empêchant les piqûres à partir de la dose de 0,3 g/l d'azadirachtine. Cet effet n'apparaîtrait pas, selon D. Dankwa (non publié), en employant des extraits de feuilles de neem.

L'effet répulsif de l'Azatin $E C^{\circledR}$ en application cutanée laisse entrevoir quelques perspectives dans le domaine de l'élevage en Afrique, notamment en assurant la protection du bétail contre les piqûres de glossines. Les tests ont été faits à partir d'une préparation commerciale contenant de l'azadirachtine mais aussi d'autres produits en raison du coût élevé de la purification. De ce fait, il est difficile de dissocier l'action de l'azadirachtine de celle des autres ingrédients contenus dans la formulation. En Australie (15), des insecticides à base d'azadirachtine sont utilisés en bains pour la lutte contre les ectoparasites du bétail (tiques, puces). Dans le cadre d'une application sur le terrain en Afrique en vue de mettre à profit l'effet répulsif, d'autres études sont à effectuer : rémanence sur le pelage en conditions tropicales, formulation appropriée, coût de l'utilisation, toxicité pour le bétail, résidus dans le lait et la viande.

\section{CONCLUSION}

Les résultats obtenus par contact tarsal ne permettent pas d'envisager l'utilisation des extraits de neem en pulvérisation directe ou par le traitement résiduel des lieux de repos des glossines (troncs et branches d'arbres) ainsi que leur emploi pour imprégnation de leurres (pièges et écrans). De plus, la rémanence dans la nature est très faible ( 3 à 5 jours) du fait d'une forte dégradation par la lumière (rayonnement ultraviolet) et par l'humidité (5). L'effet répulsif risque en outre de réduire le temps de contact de l'insecte avec le produit. Les effets faibles sur la mortalité et sur la reproduction lors d'applications topiques donnent peu d'espoir d'un usage sur le terrain. Cependant, d'autres études méritent d'être effectuées pour évaluer les effets de l'huile extraite des graines de neem sur les glossines. Néanmoins, les effets répulsifs observés laissent apparaître une utilisation possible en imprégnation du bétail. II est connu que dans certaines situations (transhumance du bétail, déplacement vers les abattoirs dans les zones infestées de glossines), l'application d'un produit répulsif peut avoir un intérêt en empêchant ou en limitant le contact glossines infectées-bétail, réduisant ainsi la transmission des trypanosomoses. Les éleveurs utilisent traditionnellement des plantes disponibles localement (extraits solubles de Cissus producta, feuilles de Sesbania aculeata) dont l'efficacité parait limitée voire faible. Les extraits de neem de par leurs effets répulsifs pourraient constituer un moyen simple de protéger localement les animaux (un arbre fournit $30 \mathrm{~kg}$ de graines donnant de 6 à $8 \mathrm{~kg}$ d'huile) et faire partie des moyens de lutte gérables par les communautés rurales.

\section{Remerciements}

Nous tenons à remercier pour leur appui et leurs conseils Mr. du Fretay (Société Calliope, Nogueres, France) et Dr Hellpap (Institut für Phytopathologie und angewandte Zoologie, Giessen, Allemagne).

\section{Bibliographie}

1. BIDMON H.J., KAUSER G., MOBUS P., KOOLMAN J., 1987. Effect of azadirachtin on blow fly larvae and pupae. In: Natural pesticides from the neem tree aud other trupical plants. Proceeding of the 3rd international Neem Conference, Nairobi, Kenya, 1986. Eschborn, Allemagne, GTZ, p. 253-271.

2. BURSELL E., 1958. The water balance of the tsetse pupae. Phil. Trans. R. Soc. (B), 241: 179.

3. CHAVAN S.R., NIKAM S.T., 1988. Investigation of alkanes from neem leaves and their mosquito larvicidal activity. Pesticides (Bombay); 22 (7): 32-33.

4. DORN A., REBEMACHER J.M., SEHN E., 1987. Effects of the azadirachtin on reproduction organs and fertility in the large milkweed bug, Oncopeltus fasciatus. In: Natural pesticides from the neem tree and other tropical plants. Proceeding of the 3rd international Neem Conference, Nairobi, Kenya, 1986. Eschborn, Allemagne, GTZ, p. 273-278.

5. ERMEL H., PAHLICH E., SCHMUTTERER H., 1987. Azadirachtin content of neem kernels from different geographical locations, and its dependance on temperature, relative humidity, and light. In: Natural pesticides from the neem tree and other tropical plants. Proceeding of the 3rd international Neem Conference, Nairobi, Kenya, 1986. Eschborn, Allcmagne, GTZ, p. 171-184.

6. GAABOUB I,A., HAYES D.K., 1984. Biological activity of azadirachtin, component of the neem tree inhibiting molting in the face fly, $M u s c a$ autumnalis De Geer (Diptera: Muscidae). Environ. Entomol., 13: 803-812.

7. GUILLET P., COZ J., SANNIER C., BARATHE J., MUSTAPHA A. PANSU M., ITARD J., 1979. Etude de la sensibilité à quelques insecticides, OMS 1998, OMS 1821, OMS 2, OMS 595 ET OMS 570 de glossines d'élevage : $G$. tachinoides, $G$. palpalis gambiensis et $G$. fuscipes fus cipes. Cah. ORSTOM, Sér. Ent. méd. Parasitol., 22 ; 81-87.

8. KOUL O., 1984. Azadirachtin: I . Interaction with the developpement of red cotton bugs. Entomol. Exp. Appl., 36: 85-88. 
9. LAVEISSIERE C., COURET D., TRAORE T., 1985. Tests d'efficacité et de rémanence d'insecticides utilisés en imprégnation sur tissu pour la lutte par piégeage contre les glossines. 1. Protocole expérimental. L'effet "knock down" des pyréthrinoïdes. Cah. ORSTOM, Sér. Ent. méd. Parasitol., 23 : 61-67.

10. MOURET J., 1983. Effets démographiques du diflubenzuron sur la mouche tsêtsé. Cah. ORSTOM, Sér. Ent. méd. Parasitol., 21 : 19-27.

11. NAQVI S.N.H., 1987. Biological evaluation of fresh extracts and some neem components with reference to abnormalities and esterase activity in insects. In: Natural pesticides from the neem tree and other tropical plants. Proceeding of the 3rd international Neem Conference, Nairobi, Kenya, 1986. Eschborn, Allemagne, GTZ, p. 315-330.

12. PHILOGENE B.J.R., ARNASON J.J., DONSKOV N., HUDON M., 1987. Intérêt de l'Azadirachta indica dans la lutte contre la pyrale de maïs In : Conférences internationales sur les ravageurs en agriculture. Paris France, ANPP, II (6): 227-235.

13. PRAHABAKER N., TOSCANO N.C., COUDRIET D.L., 1989. Susceptibility of the immature and adult stage of the sweet potatoes white fly (Homoptera: Aleyrodidae) to selected insecticides. J. Econ. Entomol., 82 983-988

MAKOUNDOU P.B., CUISANCE D., DUVALLET G., GUILLET P. Laboratory testing of the effects of a natural insecticide made with neem extracts (Azadirachta indica A. Juss) on Glossina fuscipes fuscipes Newstead, 1910 (Diptera: Glossinidae). Revue Elev. Méd. vét. Pays trop., 1995,48 (4) : 339-345

Azatin $\mathrm{EC}^{\mathbb{1}}$, an extract from neem (Azadirachta indica A. Juss) seeds, has been tested to examine its potential role in tsetse fly control by rural communities. Laboratory tests were performed on Glossina fuscipes fuscipes by topic applications or tarsal contacts. The topic application of Azatin $\mathrm{EC}^{\circledR}$ (30 $\mathrm{g}$ of azadirachtin per liter), induced a low mortality rate at higher dosages only (2.61 $\mu \mathrm{g}$ of azadirachtin/fly) in young males $($ DL 50 $=0.747 \mu \mathrm{g})$ and old gravid females (DL50 $=$ $\mathbf{2 . 5 1 6} \mu \mathrm{g}$ ). Productivity (number of pupae/female) decreased 4.5 -fold at higher dosages and the pupa weight was significantly lower at the beginning of the pupa period. These parameters did not significantly differ from control thereafter. The emergence rate was significantly lower at dosages higher than $0.261 \mu \mathrm{g} / \mathrm{fly}$. Flying and probing difficulties were observed at, and above, $0.261 \mu \mathrm{g} / \mathrm{fly}$, indicating a probable muscular physiological unbalance. However, tarsal contact with a piece of material treated with $3.9 \mathrm{~g} / \mathrm{m}^{2}$ azadirachtin had no effect on the mortality and emerging rates, or productivity, for the three periods of time tested $(1,2$ or $3 \mathrm{~min})$. A strong repellent effect has nevertheless been noticed after coating the host ear with a $0.3 \mathrm{~g} / 1$ azadirachtin solution. At this concentration, $70 \%$ and, at 3 g/l concentration, $90 \%$ of the flies did not feed on the host. As regards the results obtained, the possible use of this natural product in the field is discussed, in particular as a repellent for the protection of cattle in infested areas.

Key words : Glossina - Azadirachta indica - Insect control - Azadirachtin Antifeedants - Insecticides - Pupae - Topical application.
14. REMBOLD H., UHL M., MULLER T.II., 1987. Lffect of azadirachtin A on hormone titers during the gonadotrophic cycle of Locusta migratoria. In: Natural pesticides from the neem tree and other tropical plants. Proceeding of the 3rd international Neem Conference, Nairobi, Kenya, 1986 Eschborn, Allemagne, GTZ, p. 289-298.

15. RICE M., 1993. Devclopment of necm rescarch and industry in Australia. In: World Neem Conference, Bangalore, India, 24-28 février 1993 Rajahmundry, A.P., India, organized by the Indian Society of Tobacco Science, CTRI, p. 8-21.

16. STARK J.D., WALTER J.F., 1995. Neem oil components affect the efficacity of commercial neem insecticides. J. Agric. Food Chem., 43: 507512 .

17. WEBB R.E., HINEBAU M.A., LINDQUIST R.K., JACOBSON M.,1983. Evaluation of aqueous solution of neem seed extract against Liriomyza sativae and Liriomyza trifolii (Diptera: Agromyzidae). J. Econ. Entomol. 76: 357-361.

18. ZEBITZ C.P.W., 1984. Effect of some crude and azadirachtin-enriched neem (Azadirachta indica) seed kernel extracts on larvae of Acdes acgypti. Entomol. Exp. Appl., 35: 11-16.

MAKOUNDOU P.B., CUISANCE D., DUVALLET G., GUILLET P. Estudio en laboratorio de los efectos de un insecticida natural de extracto de "neem" (Azadirachta inidica A. Juss) sobre la Glossina fuscipes fuscipes Newstead, 1910 (Diptera : Glossinidae). Revue Elev. Méd. vét. Pays trop., 1995, 48 (4) : 339-345

Con el fin de establecer un sistema de lucha contra las glosinas, cuyo manejo esté a cargo de las comunidades rurales, se examinó el Azatin EC $^{\oplus}$, producido a base de extractos de granos de "neem" (Azadirachta indica A. Juss). Las pruebas se efectuaron sobre Glossina fuscipes fuscipes, tanto por aplicaciones tópicas como por contacto tarsal. La aplicación tópica del Azatin $\mathrm{EC}^{\circledR}$, con un contenido de la fórmula de $30 \mathrm{~g}$ de azadiractina por litro, provoca una baja mortalidad, la cual no se manifiesta sino a dosis elevadas $(2,61 \mu \mathrm{g}$ de azadiractina/mosca) en los machos jóvenes (DL50 $=\mathbf{0 , 7 4 7} \mu \mathrm{g}$ ) y las hembras adultas y grávidas (DL $50=2,516 \mu \mathrm{g}$ ). La productividad (pupas/hembra) disminuye 4,5 veces a altas dosis, con un peso de las pupas significativament inferior al inicio del estado pupal, pero con una evolución similar a la de los testigos. La tasa de eclosion disminuye significativamente a dosis superiores a $0,261 \mu \mathrm{g} /$ mosca. Las dificultades de vuelo y sondeo (piquete) se observan a partir de $0,261 \mu \mathrm{g} / \mathrm{glosina}$, lo que parece indicar una alteración de la fisiología muscular. Inversamente, el contacto tarsal sobre un tejido tratado a dosis de $3,9 \mu \mathrm{g} / \mathrm{m}^{2}$ de azadiractina no presenta ningún efecto sobre la mortalidad, la tasa de closion y la productividad, para las tres duraciones de contacto estudiadas $(1,2$ y 3 min). Se observa, por el contrario, un fuerte efecto repulsivo después de una unción de la oreja del huesped con una solución de $0,3 \mathrm{~g} / \mathrm{l}$ de azadiractina. A esta concentración, 70 p. 100 de las glosinas no se alimentan, porcentaje que alcanza 90 p. 100 a 3 g/l de concentración. Se discuten las posibles aplicaciones de campo en base a los efectos observados en este insecto hematófago, con énfasis en la posibilidad de aprovechar el efecto de repulsion para proteger el ganado de las zonas bajo riesgo.

Palabras clave : Glossina - Azadirachta indica - Control de insectos Azadirachtin - Inhibidores del apetito - Insecticidas - Pupas - Aplicación local 